\title{
Intragroup competition in public good games: The role of relative performance incentives and risk attitudes
}

\author{
Annarita Colasante $^{a}$, Aurora García-Gallego ${ }^{a}$, Nikolaos Georgantzis ${ }^{a, b}$, \\ Andrea Morone $^{a, c}$, Tiziana Temerario ${ }^{c}$ \\ ${ }^{a}$ LEE and Department of Economics, Universitat Jaume I, Castellón, Spain \\ ${ }^{b}$ Burgundy School of Business, School of Wine and Spirits Business, Dijon, France \\ ${ }^{c}$ Dipartimento di Economia, Management e Diritto d'impresa, Università degli Studi di Bari, \\ Aldo Moro, Italy
}

\begin{abstract}
We analyse a public good game with intragroup competition in which, generally but not always, the dominant strategy is to not contribute and, therefore, free-riding is the unique Nash equilibrium, not achieving Pareto efficiency. We propose a public good game setup where subjects' contributions are rewarded with different individual returns following a rank order voluntary contribution mechanism. It is found that the resulting competition for a better return significantly increases contributions. This effect is sensitive to the salience of return differences rewarding higher contributions. Furthermore, the positive effect of return differences on contribution levels depends on an individual's return-to-risk sensitivity as elicited through an independent risk elicitation task.
\end{abstract}

Keywords: cooperation, public good game, intragroup competition, risk attitude.

JEL codes: D01, D71, H41 


\section{Introduction}

A public good game (PGG, hereafter) gives rise to a social dilemma insofar it leads to a conflict between individual and collective incentives. Specifically, in the Nash equilibrium, individuals may free ride by contributing nothing to the public good, whereas it would be Pareto optimal if all individual resources were invested in the public good. An important feature in the emergence of the social dilemma is that individuals indistinctively access the public good irrespective of their contributions. On the other extreme, if individuals are granted access to the public good in shares equal to their participation to the common property, the social dilemma vanishes, provided that the net return to individual participation is positive. Therefore, appropriation of the return to one's own investment eliminates the social dilemma. However, this makes the essence of a public good disappear, trivially collapsing the context to one of a private good. We study the intermediate case arising when heterogeneous individual shares of the public resource, rather than proportional to individual contributions, depend on the ranking of individual contributions. That is, respectively, higher, intermediate and lower shares correspond to the highest, intermediate and lowest contributor.

An example in which contributors receive different shares from a public good can be found in educational systems in which a basic level of the product or service is shared by all users, while a higher willingness to pay for it gives access to better education. Another example can be found in social security systems which let you choose the level of the service, corresponding to different levels of contribution.

We analyse the role of intragroup competition in the sustainability of cooperation in the context of a PGG. In the repeated PGG implemented, intragroup competition is introduced by assigning different marginal per capita returns (MPCR, hereafter) according to each member's contribution. In particular, subjects in the same group are ranked according to their contributions, so that all subjects receive a positive share of the public good but the subject with the highest rank receives the larger share of it. While, under certain parameterizations, this context preserves the social dilemma nature of a PGG, our experimental results show that it provides an efficient incentive system for individual contributions to rise significantly as compared to the standard symmetricreturns version. As expected, the efficiency of this incentive system in raising individual contributions is sensitive to the asymmetry of individual returns resulting from the ranking of contributors. Therefore, this system is shown to be an important candidate for real-life tax policy measures in which the degree of appropriation of the common 
resource according to the ranking of contributors to the system could constitute an effective incentive for individuals to contribute more, without revealing the exact individual contributions to the public good, and, thus, preserving anonymity.

Furthermore, we analyse whether subjects' responsiveness to this incentive scheme relates to their risk attitudes. Specifically, we obtain individual data from a risk elicitation task and analyse whether taking higher risks in the presence of higher riskreturns predicts higher contributions in the intragroup competitive PGG.

The paper is organized as follows. In Section 2 we briefly review some relevant related literature. Section 3 motivates the research question and proposes the theoretical prediction of the game. In Section 4 we explain in detail the experimental design and state our main research questions. In Section 5 we elaborate the data analysis and show the main results. Section 6 concludes. The subjects' experimental instructions are in the Appendix.

\section{State of the art}

Cooperation and its sustainability in a social dilemma is a central research question in the economics research agenda. In the basic version of a PGG, it is individually more profitable not to contribute and "free ride" on the public good generated by the contributions of others. ${ }^{1}$ However, the socially desirable result (Pareto-efficient) in which all individuals obtain the highest aggregate payoff, is achieved when all subjects contribute.

Experimental research on repeated PGG has often shown that contributions start from around $50 \%$ of the endowment, declining towards zero as later periods of the session as reached. Dealing with the free-riding problem has given rise to alternative strategies like allowing communication among subjects (Isaac and Walker, 1988), introducing costly punishment (Fehr and Rockenbach, 2003; Fehr and Gatcher, 2000), allowing for voluntary participation depending on the share of defectors (Semmann et al., 2003), introducing proportional MPCR factors (Colasante and Russo, 2017; Lange et al., 2007), and other methods of heterogeneous appropriation of the public good like a lottery and an all-pay auction (Corazzini et al., 2010; Faravelli and Stanca, 2012).

Julian and Perry (1967) were the first to suggest competition (i.e. rivalry among players) as a way of solving or at least mitigating the free-rider problem. However, as

\footnotetext{
${ }^{1}$ Such is the game-theoretic prediction since 'not to contribute' is a dominant strategy in the one-shot and in the finitely repeated version of the game.
} 
Gunnthorsdottir and Rapoport (2006) suggest, the distinction of 'between' versus 'within' group competition is relevant. The literature shows good evidence of the positive correlation between inter-group competition and cooperation, and this evidence comes both from the lab (see, for example, Bornstein et al., 2002; Cárdenas and Mantilla, 2015; Böhm and Rockenbach, 2013; and Markussen et al., 2014) as well as from the field (Augenblick and Cunha, 2015; and Erev et al., 1993). ${ }^{2}$

Public good dilemmas with intergroup competition, have been analysed under the framework of step-level $\mathrm{PGG}^{3}$ (see Rapoport and Bornstein, 1987). The inquiry on the intergroup competition by using different social dilemma games leads to an important result: competition is a powerful tool to foster cooperation. Bornstein and Ben-Yossef (1994) analysed the effect of intergroup conflict in a prisoner's dilemma game and found out that cooperation is significantly higher when competition is introduced. Bornstein et al. (2002) studied the effect of intergroup competition on behaviour in the minimal-effort game ${ }^{4}$ and observed that intergroup competition improved collective efficiency. In the context of a PGG, Tan and Bolle (2007) analysed the effect of competition with and without monetary incentives and detected a stronger effect of competition with monetary rewards. Furthermore, Puurtinen and Mappes (2009) confirmed that competition fosters cooperation even in a simple one-shot game. Interestingly, the contribution by Markussen et al. (2014) introduces the novelty that subjects are asked to vote for competition. As a result, the majority of the subjects showed a preference for competition, which led in turn to a higher level of cooperation.

While the positive effect of intergroup competition on cooperation has been sufficiently documented, the effect of intragroup competition in enhancing cooperation still remains rather unexplored. One of the first examples is Falkinger's (1996) mechanism for overcoming the free-rider problem, where deviation from the mean contribution to the public good are taxed and subsidized, depending on the sign of the

\footnotetext{
${ }^{2}$ A very interesting theoretical approach deals with cooperation and aspects that are out of the scope of the present work like the heterogeneity within the group in collective contests (Nitzan and Ueda, 2018), the size of the group and the coordinated punishment (Hwang, 2017), or the group formation in heterogeneous societies (Lind, 2017).

3 "In step-level [...] public goods a funding threshold has to be reached before the good can be provided. [...] The step-level public good game differs strategically from the linear public good game. In the oneshot linear public good game, the dominant strategy is not to contribute at all. In the one-shot step-level public good game multiple Nash equilibria exist. An inefficient Nash equilibrium involves nobody contributing. There are efficient Nash equilibria in pure strategies where three of the $n$ players contribute (i.e., there are exactly enough contributions to reach the threshold)." (Schram et al. 2008).

${ }^{4}$ The group with the higher minimum won the competition and its members were paid according to the game's pay-off matrix. The members of the losing group received a zero payoff.
} 
deviation. Later, Falkinger et al. (2000) conduct a large series of experiment in order to examine the empirical properties of the mechanism, obtaining immediate and large efficiency gains, and their result is robust throughout many different experimental settings. A different type of intragroup competition is considered by Cabrera et al. (2013), where subjects are endogenously allocated between a major and a minor league according to their performance during the game. More recently, Angelovski et al. (2017) test a provision mechanism which utilizes rank competition to mitigate freeriding where groups compete via observable contributions for a larger share of the public good. They provide evidence that rank competition enhances efficiency in situations where discriminatory access to public goods is possible.

Our paper hopefully contributes to further explore intragroup competition in the context of a PGG, focusing on the size of a subject's ranking-dependent returns from the public good and the role of subjects' risk attitudes on their sensitivity to the incentive scheme.

\section{Theoretical framework}

We analyse a PGG with intragroup competition in which, under some parameterizations, the dominant strategy is to not contribute and, therefore, free-riding is the unique Nash equilibrium (NE), which diverges from the Pareto efficient outcome. Alternatively, under other parameterizations, the social dilemma disappears and the NE with full contribution of individual resources is Pareto efficient.

In order to address our research questions, we implement a standard PGG, where subjects are compensated with a higher individual marginal return if their contribution ranks them higher within their group. We consider three different MPCR values $\left(\alpha_{H}, \alpha_{M}\right.$ and $\alpha_{L}$ ) which are endogenously assigned according to subject's ranking relative to their levels of contribution. Levels $\alpha_{H}, \alpha_{M}$, and $\alpha_{L}$ correspond, respectively, to the individual maximal, average and minimal value of the public good return. In other words, the subject with the highest contribution will receive the highest individual marginal return, the intermediately ranked contributor will receive the intermediate return and the lowest contribution will receive the lowest return.

Consider the resulting generalized PGG. The payoff of individual $i$ in one period is expressed as: 


$$
\pi_{i}=e_{i}-c_{i}+\alpha_{i} \sum_{j=1}^{n} c_{j}
$$

where $e_{i}$ is the initial endowment, $c_{i}$ is the contribution individual $i$ makes to the common project, $\alpha_{i}$ is the individual marginal return that subject $i$ receives from the public good, and $n$ is the number of subjects in the group. Figure 1 shows the extensive form of a three-player PGG.

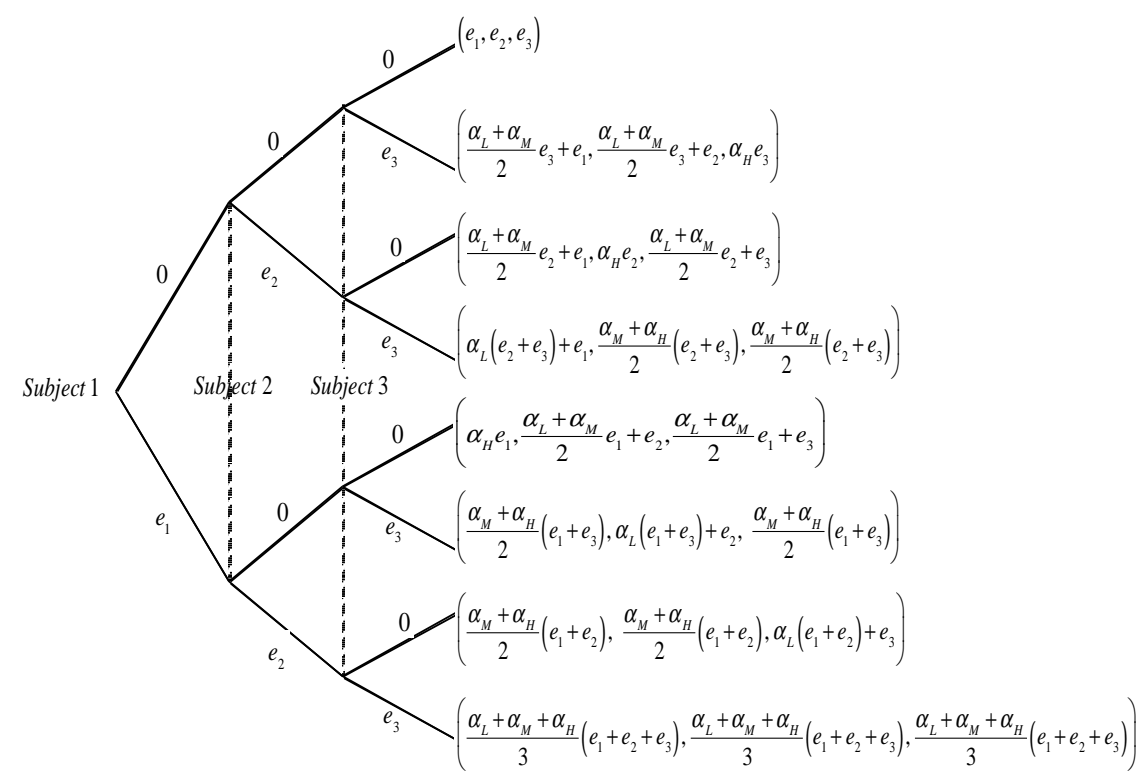

Figure 1. Example of PGG in extensive form for $n=3$ where the choices are the dominant strategies zero or full contribution

The figure shows the extensive form of the game after the iterative elimination of the first $m-1$ strictly dominated strategies. It is trivial to show that contributing any amount strictly higher than 0 and strictly lower than $e$ is dominated, respectively, by contributing zero or the full endowment. This leaves us with two possible choices: free riding (i.e., contributing zero) and full cooperation (i.e., contributing $e$ ). Solving the game by backward induction, we observe that the game is parameter dependent.

In our framework, we follow the rank order voluntary contribution mechanism. ${ }^{5}$ According to this mechanism, the individual marginal return $\alpha_{i}$ is determined by the contribution rank of individual $i$. By imposing heterogeneous MPCR and by giving the highest return to the player who contributes more, we hypothesise that, in order to get

\footnotetext{
${ }^{5}$ Angelovsky et al. (2017) use the same mechanism.
} 
the highest MPCR, the player would contribute more with respect to the condition in which the MPCR is fixed constant for all players within the group. ${ }^{6}$

We will now analyse three possible parameterizations of the game which correspond to our three treatments, named $T 0, T H$ and $T L$, and described later in Section 4. In Table 1 we specify the values of MPCR defining each treatment. Under all three parameterizations subjects' initial endowment per period is equal to 100 .

Table 1. Values of the MPCR $\alpha$ for each treatment

\begin{tabular}{|c|c|c|c|}
\hline & TO & $T H$ & $T L$ \\
\hline$\alpha_{H}$ & 0.6 & 0.9 & 0.75 \\
\hline$\alpha_{M}$ & 0.6 & 0.6 & 0.6 \\
\hline$\alpha_{L}$ & 0.6 & 0.3 & 0.45 \\
\hline
\end{tabular}

We report in Figure 2 the extensive form of the PGG under the first parameterization, i.e. $e=100$ and $\alpha_{H}=\alpha_{M}=\alpha_{L}=0.6$. This corresponds to the baseline treatment $T O$.

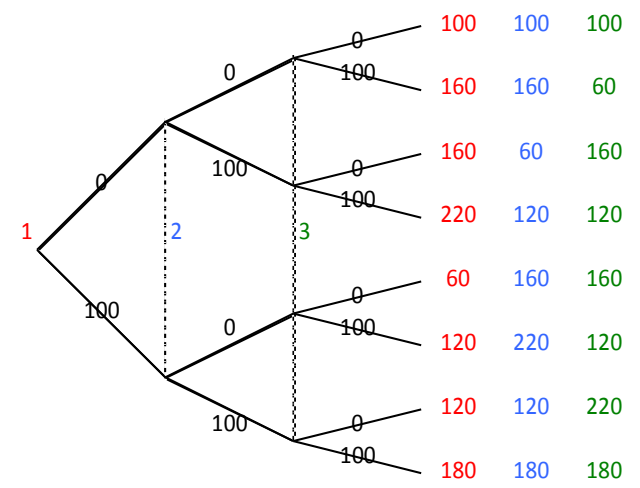

Figure 2. Extensive form of the PGG in $T 0\left(n=3, e=100, \alpha_{H}=\alpha_{M}=\alpha_{L}=0.6\right)$

Solving this game by backward induction we get the unique NE of the game in $\left(c_{1}=c_{2}=c_{3}=0\right)$, that is, all players free-ride.

\footnotetext{
${ }^{6}$ In fact, most of the previous literature regarding heterogeneous returns analyse the effect of risky or uncertain returns (see, for example, Levati and Morone, 2013 and Fischbacher et al., 2014). In such experiments, returns are both heterogeneous and unknown before the provision, as it occurs in our setting. However, returns are randomly assigned according to an (un)known probability distribution. In our setting, on the opposite, subjects have a little power to influence the MPCR they get: the more they invest, the higher the probability to get the highest return. The uncertainty related to the return, in our setting, solely depends on the others' contribution. This is why we expect that competing for a MPCR may trigger cooperation.
} 
In Figure 3 we report the extensive form of the game under the second parameterization, i.e. $e=100$ and $\alpha_{H}=0.9, \alpha_{M}=0.6, \alpha_{L}=0.3$. This corresponds to treatment $T H$.

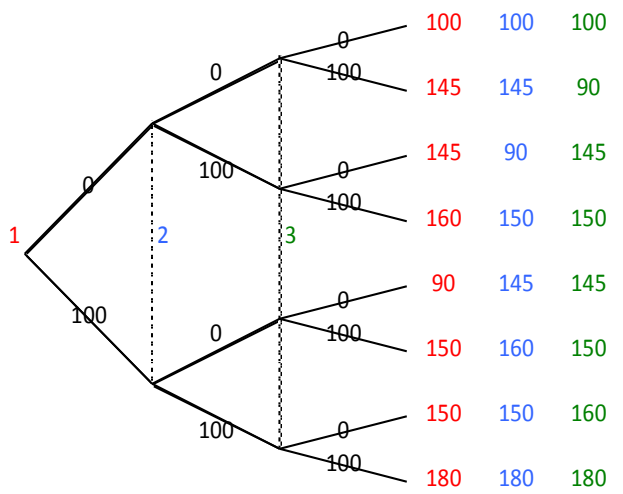

Figure 3. Extensive form of the PGG in $T H\left(n=3, e=100, \alpha_{H}=0.9, \alpha_{M}=0.6, \alpha_{L}=0.3\right)$

This version of the game has two NE in pure strategies in $\left(c_{1}=c_{2}=c_{3}=0\right)$ and $\left(c_{1}=c_{2}=c_{3}=100\right)$. Moreover, the game has one NE in mixed strategies where all players choose to free ride with probability 0.51 .

Finally, Figure 4 represents the extensive form of the game under the third parameterization, i.e. $e=100$ and $\alpha_{H}=0.75, \alpha_{M}=0.6, \alpha_{L}=0.45$. This corresponds to treatment $T L$.

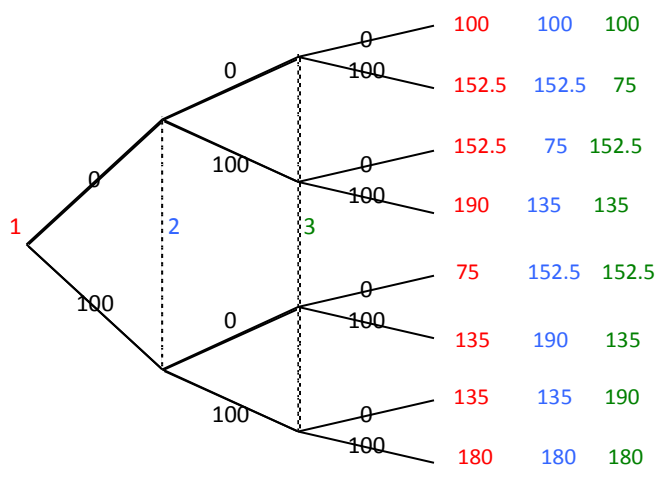

Figure 4. Extensive form of the PGG in $T L\left(n=3, e=100, \alpha_{H}=0.75, \alpha_{M}=0.6, \alpha_{L}=0.45\right)$

Solving this version of the game by backward induction we get the unique NE of the game: $\left(c_{1}=c_{2}=c_{3}=0\right)$. In this treatment, this particular parameterization of the 
incentive system is interesting since it leads to a unique NE, keeping the social dilemma nature of the game unchanged.

\section{Framework and experimental design}

\subsection{The PGG with intragroup competition}

We implement a repeated PGG with partner matching. We consider heterogeneous MPCR factors in order to generate intragroup competition. We run a control treatment $T O$ in which subjects play the standard PGG and two additional treatments, $T H$ and $T L$. In the two new treatments, subjects are ranked according to the size of their own contribution. In particular, the highest contributor gets $\alpha_{H}$ as return from the project, the second contributor gets $\alpha_{M}$ and the lowest contributor gets $\alpha_{L}$. Observe in Table 1 that treatments $T H$ and $T L$ differ in the three possible values of $\alpha$. By design, values of parameter $\alpha$ are such that in $T H$, the distance between the maximal, intermediate and the minimal values is twice the values in $T L$.

Ties are possible in all three treatments. When a tie occurs, the subjects involved get the average MPCR, computed as follows. If there are two equal contributions and a third one which is lower, the equally contributing subjects get the average between $\alpha_{H}$ and $\alpha_{M}$, and the other gets $\alpha_{L}$. In the case in which there are two equal contributions while there is one contribution higher than them, the two players with equal contributions get the average between $\alpha_{L}$ and $\alpha_{M}$ while the higher contributor gets $\alpha_{H}$. Hence, group members contributing the same amount get the same return equal to $\alpha_{M}$. Our treatments are specifically designed to answer the question whether subjects contribute more to the public good in the presence of intragroup competition. Moreover, we also test whether such an effect increases with the level of competition and whether it depends on subjects' risk attitudes.

By accounting for intragroup competition, our setting allows us to address the following research questions:

(R1) The more a group member can individually benefit from the final outcome if her contribution is the highest, the more the subject will contribute to the common project.

This conjecture leads to our second research question: 
(R2) The level of contribution is affected by the salience of the difference in individual returns rewarding a member's higher intragroup rank.

We hypothesise that the higher distance between $\alpha_{H}$ and $\alpha_{L}$ in treatment $T H$ induces a higher level of competition as compared to treatment $T L$ and, as a consequence, a higher level of contribution. Normally, investing a high percentage in a public good can be perceived as risky because it may imply a high potential loss, being this an incentive to free-ride. By introducing a competition mechanism partly reduces such a risk. Theoretically, players contributing more get a larger benefit. In a broader sense, this could be comparable to a proportional system like the one proposed by Colasante and Russo (2017) or Gunnthorsdottir and Rapoport (2006). In their experiments it emerges that, by taking a return proportional to the amount invested, cooperation is sustained. In our treatment $T H$, the maximal return is 0.9 , so that the money invested in the public good has a similar return to private consumption. Furthermore, the distance between the minimal and the maximal MPCR is 0.6 (while it is 0.3 in $T L$ ). Therefore, we hypothesise that in $T L$ subjects will have less incentive to invest high amounts in the public good, since the maximal and intermediate MPCR values are very close.

A total of 216 undergraduate students from the Universitat Jaume I, Castellón (Spain) participated in the experiment. Upon entering the laboratory, they were randomly assigned to visually isolated computer terminals. The instructions were distributed in hardcopy. ${ }^{7}$ The participants played the PGG for 10 rounds, this fact being common knowledge for subjects from the beginning of the session. Subjects got carefully informed about the anonymous group formation and matching protocol used. No opportunity was given to subjects to communicate within or between groups during the session. A session lasted up to 50 minutes and average earnings per subject were around $12 €$.

In all treatments, the decision making process in a session was as follows. In every round, each subject received an initial endowment of 100 Experimental Currency Units (ExCUs) and had to decide - privately and anonymously - how much of this endowment to allocate to a common project, the rest kept privately. Once all members of the group had submitted their decision, subjects got perfect information about the last period contribution of other players in the same group. Furthermore, subjects had information about the exact MPCRs of the specific treatment. In other words, participants in the

\footnotetext{
${ }^{7}$ See the instructions to experimental subjects in the Appendix.
} 
baseline knew that the MPCR was the same for all subjects, while the ones taking part in treatments $T H$ and $T L$ knew that the MPCR depended on the individual contribution levels and, therefore, that there was competition within the group. At the end of each period, subjects received feedback about their own payoff for that period.

\subsection{Elicitation of risk attitudes}

At the end of period 10, we elicited each subject's attitude towards risk. ${ }^{8}$ To do that, we implemented the Lottery Panel risk elicitation task, by Sabater-Grande and Georgantzis (2002), in which each subject chooses his/her preferred lottery from each panel of the four in Figure 5.

Panel 1

\begin{tabular}{|l|c|c|c|c|c|c|c|c|c|c|}
\hline Prob. & $\mathbf{1}$ & $\mathbf{0 . 9}$ & $\mathbf{0 . 8}$ & $\mathbf{0 . 7}$ & $\mathbf{0 . 6}$ & $\mathbf{0 . 5}$ & $\mathbf{0 . 4}$ & $\mathbf{0 . 3}$ & $\mathbf{0 . 2}$ & $\mathbf{0 . 1}$ \\
\hline$€$ & 1.00 & 1.10 & 1.30 & 1.50 & 1.70 & 2.10 & 2.70 & 3.60 & 5.40 & 10.90 \\
\hline Choice & & & & & & & & & & \\
\hline
\end{tabular}

Panel 2

\begin{tabular}{|l|c|c|c|c|c|c|c|c|c|c|}
\hline Prob. & $\mathbf{1}$ & $\mathbf{0 . 9}$ & $\mathbf{0 . 8}$ & $\mathbf{0 . 7}$ & $\mathbf{0 . 6}$ & $\mathbf{0 . 5}$ & $\mathbf{0 . 4}$ & $\mathbf{0 . 3}$ & $\mathbf{0 . 2}$ & $\mathbf{0 . 1}$ \\
\hline$€$ & 1.00 & 1.20 & 1.50 & 1.90 & 2.30 & 3.00 & 4.00 & 5.70 & 9.00 & 19.00 \\
\hline Choice & & & & & & & & & & \\
\hline
\end{tabular}

Panel 3

\begin{tabular}{|l|c|c|c|c|c|c|c|c|c|c|}
\hline Prob. & $\mathbf{1}$ & $\mathbf{0 . 9}$ & $\mathbf{0 . 8}$ & $\mathbf{0 . 7}$ & $\mathbf{0 . 6}$ & $\mathbf{0 . 5}$ & $\mathbf{0 . 4}$ & $\mathbf{0 . 3}$ & $\mathbf{0 . 2}$ & $\mathbf{0 . 1}$ \\
\hline$€$ & 1.00 & 1.70 & 2.50 & 3.60 & 5.00 & 7.00 & 10.00 & 15.00 & 25.00 & 55.00 \\
\hline Choice & & & & & & & & & & \\
\hline
\end{tabular}

Panel 4

\begin{tabular}{|l|c|c|c|c|c|c|c|c|c|c|}
\hline Prob. & $\mathbf{1}$ & $\mathbf{0 . 9}$ & $\mathbf{0 . 8}$ & $\mathbf{0 . 7}$ & $\mathbf{0 . 6}$ & $\mathbf{0 . 5}$ & $\mathbf{0 , 4}$ & $\mathbf{0 . 3}$ & $\mathbf{0 . 2}$ & $\mathbf{0 . 1}$ \\
\hline$€$ & 1.00 & 2.20 & 3.80 & 5.70 & 8.30 & 12.00 & 17.50 & 26.70 & 45.00 & 100.00 \\
\hline Choice & & & & & & & & & & \\
\hline
\end{tabular}

Figure 5: The Lottery Panels risk elicitation task

The risk elicitation task used here faces subjects with four decision problems. In each one of them, a panel of nine binary lotteries and a trivial one (leading to the certain gain of $1 €)$ are offered for them to choose one. In each non trivial lottery, a positive outcome entails a probability $q$ of earning $Y €$ and a probability $1-q$ to earn nothing. Each lottery panel is constructed in a way such that riskier lotteries (involving a higher

\footnotetext{
${ }^{8}$ At the beginning of the session, the instructions were read aloud and subjects were told that immediately after period 10, they would have to answer some questions that would also affect the final earnings.
} 
probability to earn nothing) have a higher expected payoff. In fact, as shown in:

$$
E V(L)=q Y=m+(1-q) t \Rightarrow Y(q)=\frac{m+(1-q) t}{q},
$$

the expected payoff is increased above the certain reward of $1 €$ by an amount which is proportional to the probability of the unfavorable outcome. The four lottery panels that we will denote as P1 to P4) correspond to different values of the risk-return parameter $t$, generating an increase in the lotteries' expected values as one moves from safer options (left side of the lottery panels) to riskier ones (right side of the lottery panels) within the same panel. In particular, the four panels of lotteries were constructed using $m=1 €$ in all lottery panels and $t=0.1,1,5$ and 10 , respectively for lottery panels P1 to P4, respectively. By the definition of the task, Expected Utility maximizers with a higher degree of risk aversion will choose weakly (due to the discreteness and the boundaries of the choice space) higher probability lotteries. It has been shown elsewhere that if they have a standard CRRA utility functions their choices will be weakly riskier as we move from P1 to P4. Given the increasing Expected Value of lotteries as the winning probability is decreased, risk neutral and risk loving subjects should choose the lowest winning probability lottery with $q=0.1{ }^{9}$

Based on a large sample $(\mathrm{N}=785)$ which was almost equally split between financially rewarded and hypothetical choices $(\mathrm{N}=401$ and $\mathrm{N}=385$, respectively), García-Gallego et al. (2012) show that over $85 \%$ of choices can be explained by two orthogonal factors. The first factor weights equally subjects' choices in the four lottery panels and can be meaningfully interpreted as a subject's aversion to risk, given that the average choice in the four lottery panels indicates a subject's unwillingness to take risks. We will approximate this Factor1 by a subject's average choice across the four lottery panels and we will use the term 'risk aversion' to refer to it. The second factor weights negatively the choices in P1 and P2 and positively the P3 and P4 choices. Furthermore, the extreme lottery panels P1 and P4 are weighted equally to each other in absolute terms and double as much as choices in P2 and P3. Therefore, the second component represents the difference in choices as we move across the most distant and across the intermediate lottery panels and can be meaningfully interpreted as the

\footnotetext{
${ }^{9}$ For more details about this task, see Attanasi et al. (2018), where meaningful alternative classifications of subjects are used based on the (non) monotonicity of choices as we move from P1 to P4. However, rather than the center of focus, in the present paper this task is used as a mere instrument for risk attitude elicitation.
} 
reaction of choices to different risk return parameters. We will approximate this component by Factor2 $=[2(\mathrm{P} 1-\mathrm{P} 4)+(\mathrm{P} 2-\mathrm{P} 3)]$ and we will refer to it with the term 'sensitivity to risk-returns'.

Regarding the effect of these components on behavior in the main experiment, and assuming that the contribution by subjects to the public good can be seen as an uncertain prospect, we can formulate our third research question:

(R3) More risk averse subjects (higher score in Factor1) should contribute less; subjects which are more sensitive to the risk return (higher score in Factor2) should contribute more, the higher the dispersion of MPCRs.

\section{Data analysis and main results}

\subsection{Descriptive statistics}

We ran two sessions for each treatment. Each session involved 36 participants. Figure 6 and Table 2 summarize our preliminary results. In comparison with the baseline, contributions are higher in the competition treatments $T H$ and $T L$. In line with previous studies, the percentage of contribution in the baseline is around $50 \%$ of the endowment in the first period, and this level slowly declines along time. Although contribution levels are the highest in $T H$ every period ${ }^{10}$, contribution in both treatments $T H$ and $T L$ show an increasing pattern. Interestingly, the highest contribution is observed in $T H$, the treatment in which we induced the highest level of competition. As a result, we confirm that not only intragroup competition fosters cooperation but, also, the degree of competition measured by the distance between the maximal and the minimal MPCR levels matters too. ${ }^{11}$ Observe in Table 2 that the difference in contribution across treatments is statistically significant; the two-sided Wilcoxon rank-sum for the comparison of average contribution at group level rejects the null hypothesis.

\footnotetext{
${ }^{10}$ The difference in contribution is statistically significant (the $p$-value is 0.01 from a two-sided Wilcoxon rank-sum test).

${ }^{11}$ In order to disentangle the effect of competition from that caused by the size of MPCR differences, we have compared these results with additional treatments, reported in a companion paper, in which heterogeneous MPCRs were randomly assigned after the contribution decision. We denote these as "risky treatments". We have found that the contribution levels when subjects compete for a higher MPCR are significantly higher than those in the risky treatments. For additional details on this, see Colasante et al. (2017).
} 


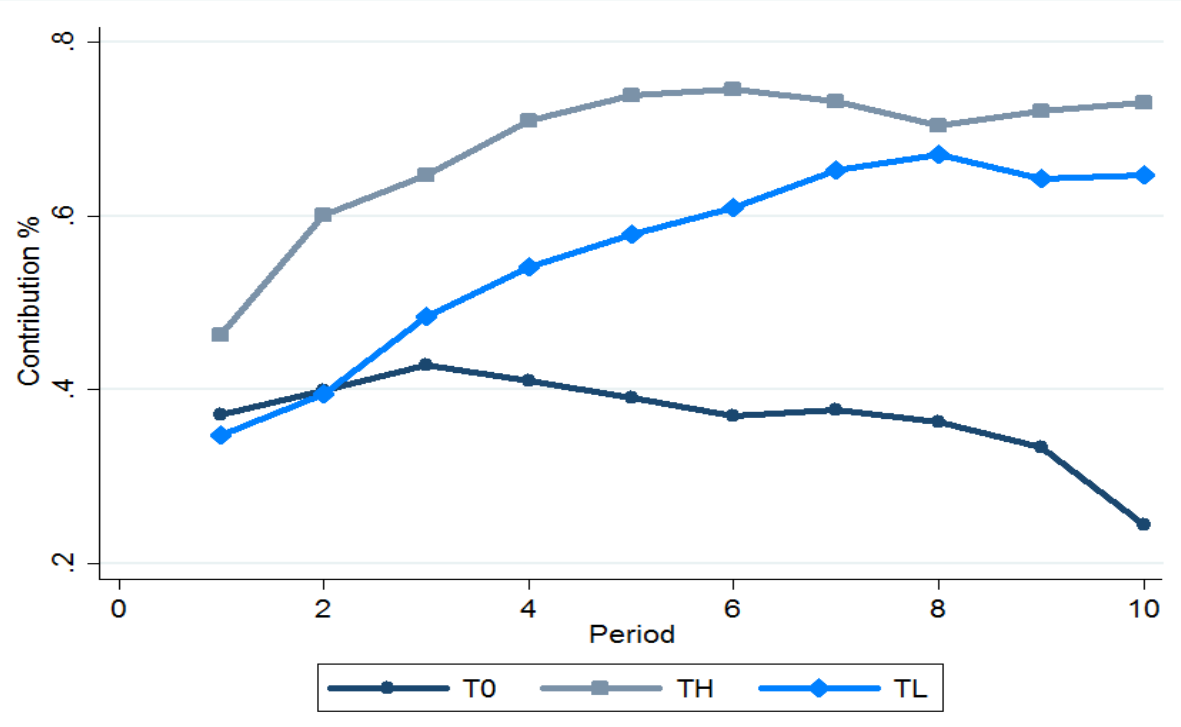

Figure 6: Average contribution (as \% of total endowment) over time

The pattern shown in Figure 6 confirms that competition fosters cooperation reflected on higher contributions at an aggregate level.

Table 2: Descriptive statistics of contribution levels

\begin{tabular}{c|ccccc|cc}
\hline & \multicolumn{5}{|c|}{ Descriptive statistics } & \multicolumn{2}{c}{ Wilcoxon test } \\
\hline Treatment & N & Group & Mean & Median & SD & $\boldsymbol{Z}$ & $\boldsymbol{p}$-value \\
\hline TO & 720 & 24 & 36.87 & 30 & 31.29 & & \\
$T H$ & 720 & 24 & 67.92 & 80 & 34.31 & -19.21 & 0.00 \\
$T L$ & 720 & 24 & 55.68 & 60 & 33.70 & -11.88 & 0.00 \\
\hline
\end{tabular}

Note: The Wilcoxon test allows for the comparison between the baseline and the two competition treatments $T H$ and $T L$ at group level.

The descriptive statistics in Table 2 highlight the differences between the baseline without competition and the two treatments with competition. Observe that $\mathrm{TH}$ is characterised by the highest mean and median contribution. One may conjecture that such evidence may imply that every subject in the group has contributed with amounts close or equal to the total endowment. However, this is contradicted by significant within-treatment heterogeneity, as indicated by the high standard deviations. To better understand whether subjects persistently contribute a high amount, we compute the 
standard deviation of the contribution in each group. In $T H$, where the distance between MPCR is higher, the heterogeneity among contributions at an individual level is exacerbated, as shown by the average standard deviation $(15.65,19.72$ and 15.70 in treatments $T 0, T H$ and $T L$, respectively). In order to analyse whether such heterogeneity is due to the existence of multiple NE characterising treatment $T H$-to invest either 0 or the whole endowment- we have extracted the share of subjects per treatment that behaved as free-rider (contributing $c_{i}=0$ ) or full contributor (contributing $\left.c_{i}=100\right)$ in each period.

In Figure 7 we represent in solid blue line the trend of the percentage of freeriders per period in each treatment, while the dashed red line describes the time evolution of the percentage of full contributors in each treatment. Observe that in the baseline $\mathrm{TO}$, the percentage of free-riders per period drastically increases along time leading to a reduction in the average contribution.

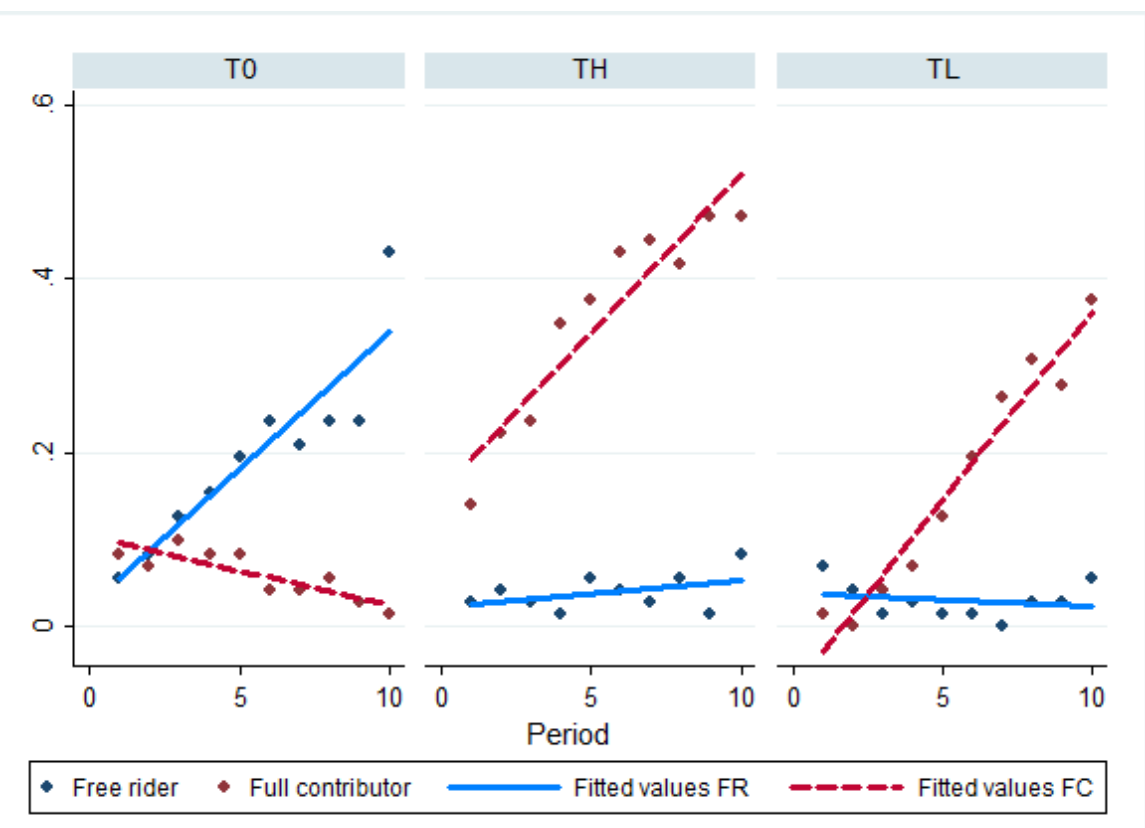

Figure 7. Share of subjects contributing either 0 or 100 in each period. Blue dots represent the share of free-riders and the blue line is the linear fit of it. Red dots represent the share of full contributors and the red dashed line is the linear fit of that share.

In $T L$ the competition mechanism only boosts the percentage of full contributors along time. Related to $T H$, a substantial increase in the percentage of full contributors is detected, reaching $50 \%$. In this treatment we observe that competition sustains high contribution levels, but also the percentage of free-riders marginally increases. An 
explanation for the increasing gap between the minimal and maximal levels of contribution registered in $T H$ over time may be found in that subjects play one of the two NE in pure strategies possible. On one hand, we find subjects that prefer to reach the highest payoff by maximising the private investment, therefore contributing nothing. On the other hand, there are subjects maximising individual payoff by trying to get the maximum MPCR choosing full contribution to the public good. To test whether our conjecture is sustainable, we have identified whether competition affects the probability of playing not to contribute at all or choosing a full contribution.

In a competitive environment, making a decision implies taking into account others' decisions. Furthermore, the individual decision to contribute is strongly affected by the MPCR received in the previous period. For example, subjects receiving the minimal MPCR in the previous period may be encouraged to free-ride in order to maximise private earnings in the current period. We hypothesize that the probability to be either a free-rider $\left(\operatorname{Pr}\left(c_{i}=0 \mid X\right)\right)$ or a full contributor $\left(\operatorname{Pr}\left(c_{i}=100 \mid X\right)\right)$ may be described by the following formula:

$$
\begin{aligned}
& \operatorname{Pr}\left(c_{i}=0 \mid X\right)=\text { Const }+\beta_{0} \text { rank }_{i t-1}+\beta_{1} \text { others }_{i t-1}+T H+T L+\varepsilon \\
& \operatorname{Pr}\left(c_{i}=100 \mid X\right)=\text { Const }+\beta_{0} \text { rank }_{i t-1}+\beta_{1} \text { others }_{i t-1}+T H+T L+\varepsilon
\end{aligned}
$$

where rank $_{i-1}$ denotes is the lagged ranking assigned to each subject according to his/her level of contribution (i.e., the subject with the highest contribution receives rank=1 which corresponds to a MPCR of 0.9 in treatment $T H$ and of 0.75 in $T L$ ). The variable others $s_{i t-1}$ represents the lagged value of the aggregate contribution of all other subjects in the same group. Finally, $T H$ and $T L$ are treatment dummies.

As displayed in Table 3, being a participant in treatment $T H$ (treatment $T L$ ) significantly increases (decreases) the probability to be a full contributor (free-rider). Notice that, far from expected, the reduction in the probability to free-ride is smaller in treatment $T H$-where competition is stronger- than in $T L$. Such an evidence constitutes a first support to our conjecture about the existence of multiple NE in $T H$. The main difference between results in models (1) and (2) of Table 3 is found in the role played by the variable $\operatorname{rank}_{i t-1}$, that is significant only for the probability of becoming a freerider. In an indirect way, the MPCR received in the previous period becomes significant. On the contrary, the ranking in the previous period does not affect the willingness to choose full contribution. 
Table 3. Panel probit regression models

\begin{tabular}{lcc}
\hline & $(1)$ & $(2)$ \\
\hline & $\operatorname{Pr}(c=0 \mid X)$ & $\operatorname{Pr}(c=100 \mid X)$ \\
rank $_{i t-1}$ & $0.16^{* *}$ & -0.07 \\
& $(0.07)$ & $(0.05)$ \\
others $_{i t-1}$ & $-0.01^{* * *}$ & $0.02^{* * *}$ \\
& $(0.00)$ & $(0.00)$ \\
TH & $-0.89^{* * *}$ & $1.66^{* * *}$ \\
& $(0.32)$ & $(0.39)$ \\
$T L$ & $-1.68^{* * *}$ & $0.821^{* *}$ \\
& $(0.36)$ & $(0.391)$ \\
Constant & $-1.05^{* * *}$ & $-4.95^{* * *}$ \\
& $(0.28)$ & $(0.44)$ \\
& & \\
Observations & 1,944 & 1,944 \\
Number of subjects & 216 & 216 \\
\hline Standard errors in parentheses & \\
$* * * p<0.01, * * p<0.05, * p<0.1$ &
\end{tabular}

Others' contribution, on aggregate, has an effect in both dependent variables, although the impact is stronger on the probability of playing full contribution.

To wrap up, the competition mechanism turns out to be an effective tool not only for increasing but also for sustaining full contribution within the group. However, the allocation of heterogeneous MPCRs according to the individual contribution leads to a controversial effect: there is a proportion of subjects who prefer not to take part in the competition but simply taking advantage by free-riding. This happens mostly in treatment $T H$, where both free-riding and full cooperation are NE.

\subsection{Determinants of the contribution level}

So far, we have shown that the existence of competition within subjects of the same group triggers cooperation. To better understand what are the main determinants of individual contributions, and to check whether risk preferences play a role in the investment decision, we run a mixed panel regression. We consider as a dependent variable the individual contribution, and observations are clustered at group level. The main explanatory variables that may have an effect on the individual contributions are: treatment dummies for $T H$ and $T L$, and $\operatorname{rank}_{i t-1}$, the individual (lagged) rank, assigned according to the subject's contribution. Furthermore, the variable Factor $1_{i}$ is the average value of subject $i$ choices in the four lottery panels, that is, $(\mathrm{P} 1+\mathrm{P} 2+\mathrm{P} 3+\mathrm{P} 4) / 4$. This factor is a proxy of the individual risk aversion, that is, the higher its value the 
more risk averse the subject is. ${ }^{12}$ Factor $2_{\mathrm{i}}$ is computed as $[2 *(\mathrm{P} 1-\mathrm{P} 4)+(\mathrm{P} 2-\mathrm{P} 3)]$, and measures subject $i$ 's willingness to vary his/her choices as we move from lottery panel P1 to P4. It can be interpreted as the reaction of choices to different risk return parameters and we will refer to it as sensitivity to risk-returns. In order to verify whether there are significant differences among treatments, we add to our model the interaction of the aforementioned variables with the treatment dummies. Results of the model as well as of the nested models are shown in Table 4.

Table 4. Linear mixed panel regression models with individual contribution as the dependent variable

\begin{tabular}{|c|c|c|c|c|}
\hline & (1) & (2) & (3) & (4) \\
\hline$T H$ & $\begin{array}{c}33.48 * * * \\
(6.93)\end{array}$ & $\begin{array}{c}72.01 * * * \\
(7.14)\end{array}$ & $\begin{array}{c}71.52 * * * \\
(7.21)\end{array}$ & $\begin{array}{c}87.93 * * * \\
(5.31)\end{array}$ \\
\hline$T L$ & $\begin{array}{l}21.16 * * * \\
(6.93)\end{array}$ & $\begin{array}{c}45.61 * * * \\
(7.10)\end{array}$ & $\begin{array}{c}44.93 * * * \\
(7.17)\end{array}$ & $\begin{array}{c}44.22 * * * \\
(5.37)\end{array}$ \\
\hline $\operatorname{rank}_{\mathrm{it}-1}$ & $\begin{array}{c}-3.087 * * * \\
(0.44)\end{array}$ & $\begin{array}{l}-0.39 \\
(0.39)\end{array}$ & $\begin{array}{l}-0.28 \\
(0.41)\end{array}$ & $\begin{array}{l}-0.08 \\
(0.66)\end{array}$ \\
\hline $\operatorname{rank}_{\mathrm{it}-1} * T H$ & & $\begin{array}{c}-12.84 * * * \\
(0.59)\end{array}$ & $\begin{array}{c}-13.03 * * * \\
(0.60)\end{array}$ & $\begin{array}{c}-13.49 * * * \\
(1.00)\end{array}$ \\
\hline $\operatorname{rank}_{\mathrm{it}-1} * T L$ & & $\begin{array}{c}-8.15 * * * \\
(0.52)\end{array}$ & $\begin{array}{c}-8.15 * * * \\
(0.55)\end{array}$ & $\begin{array}{c}-7.01 * * * \\
(0.87)\end{array}$ \\
\hline Factor $1_{i}$ & & & $\begin{array}{c}1.72 \\
(2.43)\end{array}$ & $\begin{array}{c}7.21 \\
(5.26)\end{array}$ \\
\hline Factor $2_{i}$ & & & $\begin{array}{l}2.53 * \\
(1.30)\end{array}$ & $\begin{array}{c}-8.24 * * \\
(3.27)\end{array}$ \\
\hline Factor $1_{i} * T H$ & & & & $\begin{array}{c}-15.37 * * \\
(7.39)\end{array}$ \\
\hline Factor $1_{\mathrm{i}} * T L$ & & & & $\begin{array}{c}6.66 \\
(8.38)\end{array}$ \\
\hline Factor $2_{\mathrm{i}} * T H$ & & & & $\begin{array}{c}13.98 * * * \\
(5.39)\end{array}$ \\
\hline Factor $1_{\mathrm{i}} * T L$ & & & & $\begin{array}{c}2.64 \\
(4.89)\end{array}$ \\
\hline Constant & $\begin{array}{c}46.10 * * * \\
(5.07)\end{array}$ & $\begin{array}{c}38.02 * * * \\
(5.03) \\
\end{array}$ & $\begin{array}{c}37.20 * * * \\
(5.23)\end{array}$ & $\begin{array}{c}25.14 * * * \\
(3.64) \\
\end{array}$ \\
\hline Observations & 1,944 & 1,944 & 1,944 & 1,944 \\
\hline $\begin{array}{l}\text { Number of groups } \\
\text { Wald }\end{array}$ & $\begin{array}{c}72 \\
\chi^{2}=73.32 * * *\end{array}$ & $\begin{array}{c}72 \\
\chi^{2}=792.61 * * *\end{array}$ & $\begin{array}{c}72 \\
\chi^{2}=740.27 * * *\end{array}$ & $\begin{array}{c}72 \\
\chi^{2}=693.63 * * *\end{array}$ \\
\hline
\end{tabular}

Observations are clustered at group level

Standard errors in parentheses

$* * * p<0.01, * * p<0.05, * p<0.1$

\footnotetext{
${ }^{12}$ Individual risk preferences were elicited straight after the experiment. In order to check whether the experiment may have influenced risk attitudes, we run a propensity score matching test comparing results in terms of Factor1. For the test we have used our experimental data and other sample in which the risk task was performed before the experiment. As a result, we have found no significant differences. The additional data sample and the results of the test are available from the authors upon request.
} 
The positive and significant coefficients relative to the treatment dummies confirm that individual contribution is higher when competition is introduced, as shown in Figure 6. This result is robust to all the specifications considered. The coefficient relative to the rank (i.e. $\operatorname{rank}_{i t-1}$ ), is negative ${ }^{13}$ and significant in model (1) of this table. In the model specifications (2) to (4), the effect on contribution is captured by the interacted variable rank with the treatment dummies: the impact of past MPCR, indirectly measured by the variable, is stronger in treatment $T H$. This suggests that, in general, competition generates a virtuous circle: contributing more results in the highest MPCR and, as a result, the subject is willing to contribute more in order to maintain the same benefit.

Concerning risk aversion, observe that Factor $1_{i}$ has no significant impact on individual contribution levels, whereas the sensitivity to risk-returns positively affects the willingness to contribute. In other words, the more a subject makes riskier choices when faced with higher returns to riskier options, the more the subject is likely to contribute in absolute terms. When introducing the interaction between risk aversion measures and the treatment dummies (see model (4) in the table), interesting second order effects emerge. Factor $1_{i}$ turns out to be significant when interacted with the $T H$ dummy, which means that, in a context with high uncertainty about the MPCR, risk averse subjects are less willing to contribute. Regarding the sensitivity to risky returns (measured by Factor $2_{\mathrm{i}}$ ), observe in model (3) that the higher the propensity to take higher risks as we move from panel $\mathrm{P} 1$ to $\mathrm{P} 4$, the higher the propensity to take the risk of contributing more. In model (4), the interaction of Factor $2_{\mathrm{i}}$ with the treatment dummy variable $T H$ is positive and significant. Specifically, the effect of the corresponding interaction coefficient indicates that a subject's sensitivity to risky returns becomes more significant in explaining contributions as we move from TO to TH.

As a result, the estimates of the econometric models in Table 4 answer the research questions. In fact, both $T H$ and $T L$ dummy coefficients are significant at $1 \%$, indicating that the presence of intragroup competition for a higher individual return of the public good increases individual contributions in comparison to the levels observed in the baseline where returns are not linked to a group member's relative contribution

\footnotetext{
${ }^{13}$ Notice that a value of 1 for variable rank is assigned to the subject with the highest contribution. This means that the higher the contribution the lower the value of the rank variable.
} 
(R1). Moreover, the size of the aforementioned dummy coefficients indicates that the effect of competition for a higher individual return is sensitive to the magnitude of the difference between individual return factors (R2). Therefore, competition matters more the higher is the ex post asymmetry in individual returns induced by the relative ranking of individual contributions. The sensitivity to the magnitude of the ex post asymmetry is especially interesting when such individual return differences are used as an incentive for the members of the group to increase their contributions. Our results show that increasing the ex post asymmetry is an efficient instrument leading to collectively better outcomes.

Taking into account subjects' risk preferences, a possible explanation for our main results may be that the effect of competition for a higher in-group rank is risk attituderelated, and is driven by subjects' heterogeneous degrees of sensitivity to variations in the return to risk, as suggested by our third research question (R3).

\section{Conclusions}

The purpose of this paper has been to investigate whether intragroup competition enhances subjects' contributions to a public good. In order to test the impact of intragroup competition on the level of cooperation, we have revisited the standard PGG (TO) and add two new treatments $(T H, T L)$ in which three different values of the MPCR are assigned to group members so that subjects are ranked according to the size of their own contribution to the public good. The two treatments $T H$ and $T L$ include competition within a group and differ only in the "degree of competition". In particular, in treatment $\mathrm{TH}$ the distance between the minimal and the maximal values of the MPCR is equal to 0.6 , exactly twice the difference in treatment $T L$.

Our results confirm our three research questions. Specifically, the more each group member can individually benefit from the final outcome, the more the subject contributes to the common project. Furthermore, the individual level of contribution is affected by the salience of differences among individual returns according to the member's intragroup ranking, thus resulting in a significantly higher level of contributions in treatment $\mathrm{TH}$.

Finally, we have shown that our results are related to the subject's proneness to take higher risks when motivated by higher returns to risk. We have elicited individual 
risk preferences through the Lottery Panels risk elicitation task by Sabater and Georgantzis (2002). We have computed what we call Factor1, the average choices in the four panels; and Factor2 which is the sensitivity to risky-returns. We find out that more risk averse subjects contribute less, and subjects who are more sensitive to the risk return contribute more the higher the dispersion of MPCRs.

Our experimental results contribute to the understanding of how mechanisms in which returns from the public good are commensurate with the amount of resources individually invested result in higher contributions and sustainability of resources. 


\section{Aknowledgements}

Comments and discussion by participants at the NYUAD Workshop (Abu Dhabi, December 2018), at the Workshop on Empirical Behavioural Economics (Alicante, December 2017), and at the SEET meeting (Lecce, Italy, February 2018) are gratefully acknowledged. We are grateful to the Spanish Ministerio de Economía $y$ Competitividad (grant ECO2015-68469-R) and the Universitat Jaume I (grant UJIB2018-76) for financial support.

\section{References}

Angelovski, A., Neugebauer, T. and Servátka, M. (2017). Rank-order competition in the voluntary provision of impure public goods. Luiss Dipartamento di Economia\&Finanza, Working Paper 05/2017.

Augenblick, N. and Cunha, J.M. (2015). Competition and cooperation in a public goods game: A field experiment. Economic Inquiry, 53(1), 574-588.

Attanasi, G., Georgantzis, N., Rotondi, V. and Vigani, D. (2018). Lottery- and surveybased risk attitudes linked through a multi-choice elicitation task. Theory and Decision, 84(3), 341-372.

Böhm, R., and Rockenbach, B. (2013). The inter-group comparison intra-group cooperation hypothesis: comparisons between groups increase efficiency in public goods provision. PloS One, 8(2), e56152. https://doi.org/10.1371/journal.pone.0056152

Bornstein, G. and Ben-Yossef, M. (1994). Cooperation in intergroup and single-group social dilemmas. Journal of Experimental Social Psychology, 30(1), 52-67.

Bornstein, G., Gneezy, U. and Nagel, R. (2002). The effect of intergroup competition on group coordination: An experimental study. Games and Economic Behavior 41(1), 1-25.

Cabrera, S., Fatás, E., Lacomba, J. A., and Neugebauer, T. (2013). Splitting leagues: promotion and demotion in contribution-based regrouping experiments. Experimental Economics, 16(3), 426-441.

Cárdenas, J.C., and Mantilla, C. (2015). Between-group competition, intra-group cooperation and relative performance. Frontiers in Behavioral Neuroscience, 9. https://doi.org/10.3389/fnbeh.2015.00033 
Colasante, A., García-Gallego, A., Morone, A., and Temerario, T. (2017). The utopia of cooperation: does intra-group competition drive out free riding? Working Papers 2017/08, Economics Department, Universitat Jaume I, Castellón (Spain).

Colasante, A. and Russo, A. (2017). Voting for the distribution rule in a public good game with heterogeneous endowments. Journal of Economic Interaction and Coordination, 12(3), 443-467. https://doi:10.1007/s11403-016-0172-1

Corazzini, L., Faravelli, M., and Stanca, L. (2010). A prize to give for: An experiment on public good funding mechanisms. The Economic Journal, 120(547), 944-967.

Erev, I., Bornstein, G., and Galili, R. (1993). Constructive intergroup competition as a solution to the free rider problem: A field experiment. Journal of Experimental Social Psychology, 29(6), 463-478.

Falkinger, J. (1996). Efficient private provision of public goods by rewarding deviations from average. Journal of Public Economics, 62, 413-422.

Falkinger, J., Fehr, E., Gachter, S. and Winter-Ebmer, R. (2000). A simple mechanism for the efficient provision of public goods: Experimental evidence. American Economic Review, 90, 247-264.

Faravelli, M., and Stanca, L. (2012). Single versus multiple-prize all-pay auctions to finance public goods: An experimental analysis. Journal of Economic Behavior and Organization, 81(2), 677-688.

Fehr, E., and Gachter, S. (2000). Cooperation and punishment in public goods experiments. American Economic Review, 90(4), 980-994.

Fehr, E., and Rockenbach, B. (2003). Detrimental effects of sanctions on human altruism. Nature, 422(6928), 137-140.

Fischbacher, U., Schudy, S., and Teyssier, S. (2014). Heterogeneous reactions to heterogeneity in returns from public goods. Social Choice and Welfare, 43(1), 195-217.

García-Gallego, A., Georgantzis, N., Jaramillo-Gutiérrez, A. and Parravano, M. (2012). The lottery-panel task for bi-dimensional parameter-free elicitation of risk attitudes. Revista Internacional de Sociología, 70(1), 53-72.

Gunnthorsdottir, A., and Rapoport, A. (2006). Embedding social dilemmas in intergroup competition reduces free-riding. Organizational Behavior and Human Decision Processes, 101(2), 184-199.

Hwang, S-H. (2017). Conflict technology in cooperation: The group size paradox revisited. Journal of Public Economic Theory, 19, 875-898. 
Isaac, R. M., and Walker, J. M. (1988). Communication and free-riding behavior: The voluntary contribution mechanism. Economic inquiry, 26(4), 585-608.

Julian, J. W., and Perry, F. A. (1967). Cooperation contrasted with intra-group and inter-group competition. Sociometry, 30(1), 79-90.

Lange, A., List, J. A., and Price, M. K. (2007). Using lotteries to finance public goods: Theory and experimental evidence. International Economic Review, 48(3), 901927.

Levati, M. V., and Morone, A. (2013). Voluntary contributions with risky and uncertain marginal returns: the importance of the parameter values. Journal of Public Economic Theory, 15(5), 736-744.

Lind, J.T. (2017). Group structure and public goods provision in heterogeneous societies. Journal of Public Economic Theory, 19(2), 377-408.

Markussen, T., Reuben, E. and Tyran, J.R. (2014). Competition, cooperation and collective choice. The Economic Journal, 124(574), F163-F195.

Nitzan, S., Ueda, K. (2018). Selective incentives and intragroup heterogeneity in collective contests. Journal of Public Economic Theory, 20, 477-498.

Puurtinen, M. and Mappes, T. (2009). Between-group competition and human cooperation. Proceedings of the Royal Society of London B: Biological Sciences, 276(1655), 355-360.

Rapoport, A. and Bornstein, G. (1987). Intergroup competition for the provision of binary public goods. Psychological Review, 94, 291-299.

Sabater, G. and Georgantzis, N. (2002). Accounting for risk aversion in repeated prisoners' dilemma games: An experimental test. Journal of Economic Behavior and Organization, 48, 37-50.

Schram, A., Offerman, T. and Sonnemans, J. (2008). Explaining the comparative statics in step-level public good games. Handbook of Experimental Economics Results, $1,817-824$.

Semmann, D., Krambeck, H-J and Milinski, M. (2003). Volunteering leads to rockpaper-scissors dynamics in a public goods game. Nature, 425(6956), 390-393.

Tan, J.H.W. and Bolle, F. (2007). Team competition and the public goods game. Economics Letters, 96(1), 133-139. 
Appendix. Experimental instructions (translated from the original in Spanish)

\section{Treatment T0 (Baseline)}

Thank you very much for being here. The instructions are identical to all participants. Read them carefully. If you have any questions or concerns, please raise your hand and we will answer your questions individually. During the session, it is strictly forbidden to communicate with the other participants.

The unit of experimental money will be the ExCU (Experimental Currency Unit), where $100 \mathrm{ECU}=€ 10$. At the end of the session one of your decisions will be randomly chosen. Note that all choices are equally likely. The experimental payoff corresponding to the selected decision will be calculated, converted to Euros, and paid to you (privately) in cash.

\section{The Experiment}

The experiment consists of 10 independent periods in which you will interact with 2 other participants in the session. The 3 of you form a group that will remain THE SAME in all periods. The identity of the participants of your group will not be revealed to you at all during the session.

At the beginning of each period, each participant receives an endowment of 100 ECU. In any period, each member of a group has to take a decision.

Every period, you have to decide how much of your endowment you want to contribute to a common project. Your contribution decision must be not smaller than 0 ECU and not greater than 100 ECU. Furthermore, it must be an integer number. Whatever you do not contribute, you keep it for yourself ("ECU you keep").

In every period, your earnings consist of two parts:

(1) the "ECU you keep"= [100 - your contribution $]$ ECU;

(2) the "income from the project". 
The "income from the project" is calculated by adding up the contributions of the 3 members of your group and multiplying the resulting sum by a number that we call $\alpha$. That is:

Income from the project $=[$ Your contribution + Your partners' contribution $] \times \alpha$ The multiplier $\alpha$ is equal to 0.6. [TH: The multiplier $\alpha$ can be either 0.9 or 0.6 or 0.3 , where each value is equally likely.] [TL: The multiplier $\alpha$ can be either 0.75 or 0.6 or 0.45 , where each value is equally likely.].

You have to decide about your contribution without knowing the value of $\alpha$.

The income from the project is determined in the same way for every member of a group; this means that you all receive the same income from the project, regardless of the size of your individual contributions.

[TH: The income from the project is determined as follows: members in the group will be ranked in accordance to the size of their individual contributions. The highest contributor gets $\alpha=0.9$ [TL: $\alpha=0.75]$ as return from the project; the second contributor gets $\alpha=0.6$ and the lowest contributor gets $\alpha=0.3$ [TL: $\alpha=0.45$ ]. If more than one member makes the same contribution (that is, they are equally ranked), the return per capita will be calculated as follows:

- If all three members make the same contribution, each receives $\alpha=(0.9+0.6+0.3) / 3=0.6[T L: \alpha=(0.75+0.6+0.45) / 3=0.6]$

- If two members make the highest contribution (they both are ranked as first), they both receive $\alpha=(0.9+0.6) / 2=0.75$ [TL: $\alpha=(0.75+0.6) / 2=0.675]$ while the third member receives $\alpha=0.3$ [TL: $\alpha=0.45$ ];

- If two members make the lowest contribution (they are ranked as third), they receive $\alpha=(0.6+0.3) / 2=0.45$ [TL: $\alpha=(0.6+0.45) / 2=0.525]$ and the first contributor receives $\alpha=0.9$ [TL: $\alpha=0.75]$ ].

EXAMPLE: If the sum of the contributions of the three members is 60 ECU, each member receives an income from the project equal to $(0.6 \times 60)=36 \mathrm{ECU}$. 
[Treatment H: EXAMPLE: If the sum of the contributions of the three members is 60 ECU, the contributor ranked as first receives an income from the project of $(0.9 \times$ $60)=54 \mathrm{ECU}[T L:(0.75 \times 60)=45 \mathrm{ECU}]$; the second receives $(0.6 \times 60)=36 \mathrm{ECU}$ and the third $(0.3 \times 60)=18$ ECU $[T L:(0.45 \times 60)=27$ ECU $]$. However, for instance:

- If all the members are equally ranked they receive $(0.6 \times 60)=36$ ECU per capita;

- If two members are ranked as first, they both receive $[(0.9+0.6) / 2 \times 60]=45$ ECU $[T L:[(0.75+0.6) / 2 \times 60]=0.67 \times 60=40.2 \mathrm{ECU}]$ per capita; the third receives $(0.3 \times 60)=18 \mathrm{ECU}[T L:(0.45 \times 60)=27 \mathrm{ECU}]$;

- If two members are ranked as third, they both receive $[(0.6+0.3) / 2 \times 60]=27$ ECU [TL: $(0.6+0.45) / 2 \times 60=0.525 \times 60=31.5$ ECU] per capita; the highest receives $(0.9 \times 60)=54 \mathrm{ECU}[T L:(0.75 \times 60)=45 \mathrm{ECU}]$.

At the end of each period you will receive information about the contribution of your partners and your corresponding period-earnings in that period.

Before the experiment starts, you will have to answer some control questions to verify your understanding of the rules of the experiment.

Please remain seated quietly until the experiment starts. If you have any question, please raise your hand. 